\title{
Fenómenos de vacilación, sus contextos léxicos y sintácticos en entrevistas formales de legisladores a ciudadanos en el Congreso de la Ciudad de México Lexical and syntactic contexts of hesitation phenomena in formal deputy-citizen interviews conducted at Mexico City congress
}

Luis Bernardo Quesada Nieto

The Graduate Center. City University of New York

lquesadanieto@gradcenter.cuny.edu

Original recibido: 2019/04/04

Dictamen enviado al autor: 2019/09/03

Aceptado: 2020/04/23

\section{Resumen}

El presente trabajo, resultado de un análisis de corte etnográfico, tiene como propósito ofrecer una aproximación al contenido léxico y los entornos sintácticos de algunos fenómenos de vacilación (sonidos breves, repeticiones, sonidos largos, alargamientos de palabra, palabras cortadas y frases cortadas), identificados en una muestra extraída de un corpus constituido por entrevistas realizadas por un grupo de legisladores del Congreso de la Ciudad de México a aspirantes ciudadanos a ocupar el cargo de ombudsperson de la Comisión de Derechos Humanos de esa ciudad. A partir de una descripción léxico-sintáctica se exponen algunas reflexiones sobre su valor lingüístico y comunicativo, y se propone una interpretación de sus patrones de ocurrencia en las respuestas a preguntas hechas en estas entrevistas formales, basada en su grado de planificación discursiva, en la clase 
de palabra que interactúa con dichos fenómenos y en el concepto de repertorio según ha sido empleado en la teoría de translanguaging. Hacia las conclusiones se observa que los fenómenos estudiados y su distribución están directamente relacionados con palabras de la clase abierta, y con el esfuerzo cognitivo de producir mensajes gramaticales, precisos y socialmente apropiados.

Palabras clave: vacilaciones; planificación discursiva; lengua oral; clases de palabras; repertorio; translanguaging.

\section{Abstract}

This article, which is an outcome of an ethnographic research, aims to offer an insight into lexical and syntactic contexts of some hesitation phenomena (short fillers, repetitions, long fillers, word lengthening, unfinished words and unfinished phrases), identified in a corpus sample that consists of structured interviews conducted by a group of deputies of Mexico City Local Congress with citizens who applied for the ombudsperson's position at the city's Human Rights Office (Comisión de Derechos Humanos de la Ciudad de México). Drawing upon a lexical and syntactic description, some remarks on the hesitation phenomena's linguistic and communicative values are presented. I propose an interpretation of hesitation occurrence patterns that appear in the respondent's answers. This interpretation is based on the discursive planning level, the interaction between hesitation markers and word classes, and the concept of repertoire as it has been used in the theory of translanguaging. Towards the end of the manuscript I argue that the studied phenomena and their distribution are directly related to open class words, and the cognitive effort of producing grammatical, accurate and socially appropriate messages.

Keywords: hesitation markers; discursive planning; oral language; word classes; repertoire; translanguaging theory. 


\section{INTRODUCCIÓN}

Si bien hasta la fecha ha habido aproximaciones al estudio de los "fenómenos de vacilación” en español (Ávila 1999; Jeong 2011), estas aún constituyen un objeto relativamente inexplorado por las ciencias del lenguaje; existen investigaciones que han abordado algunas ocurrencias fonéticas similares a las que aquí se tratan, pero con funciones discursivas distintas (ver por ejemplo Fant 1996). Un acercamiento especializado a estos fenómenos resulta necesario para conformar rasgos de la oralidad como objeto de atención prescriptivista, históricamente valorados negativamente por un conjunto de ideas sobre la fluidez oral (Quesada 2019). Estas valoraciones negativas hacia los fenómenos de vacilación ${ }^{1}$ han provocado un gran escrutinio y una gran atención normativa en la lengua oral, que es posible rastrear en libros de texto para escolarización, guías, manuales y otros materiales que hacen énfasis en "el bien hablar" o "el buen decir", que plantean restringir los fenómenos de vacilación, disfrazarlos o eliminarlos de la lengua. La presencia persistente de dispositivos y mensajes normativos que centran la atención en este aspecto particular de la lengua oral, tanto como la larga tradición de control de la lengua y su uso de la que estas instancias normativas son resultado, ha participado y participa en la naturalización o normalización social de ese conjunto de ideas sobre la fluidez y el "habla vacilante", y ha contribuido también a crear expectativas sociales hacia determinados usos lingüísticos

1 Definidos por ahora como elementos producidos por el propio hablante, y que "alteran" o "transgreden" la fluidez verbal (ver $\$ 3.1$ infra). 
en el nivel diafásico, constituyendo expresiones de higiene verbal (Cameron 1995) que atraen la atención con especial fuerza cuando aparecen en situaciones de habla pública, sobre todo las de tipo formal o protocolar, como las interacciones oficiales que de forma cotidiana toman lugar en un parlamento o congreso, como es el caso que aquí se estudia.

El estudio de los fenómenos de vacilación es de interés para las ciencias del lenguaje, además, pues contribuye a comprender la complejidad de las relaciones entre lengua oral y escrita, sobre todo cuando la segunda es tomada como modelo de lengua. En este marco, este trabajo pretende reportar algunos hallazgos de un estudio sobre este tipo de rasgos orales y se propone realizar una aportación al estudio de esa relación entre lengua oral y escrita desde el nivel lingüístico, dejando para una discusión posterior los aspectos históricos e ideológicos del tema. ${ }^{2}$

El presente trabajo busca contribuir también a la comprensión de procesos cognitivos de planificación enunciativa, partiendo de algunas líneas

2 Consideramos que una mejor comprensión de la complejidad entre la lengua oral y la escrita debe tomar en cuenta las distintas relaciones de poder que vinculan a una y otra. Una profundización posterior se plantea por lo tanto la incorporación de una perspectiva glotopolítica de análisis (Del Valle 2017), es decir, las discrepancias o las “tensiones” que existen entre una/s forma/s de habla inclinadas o "influenciadas" por la lengua escrita y/o literaria y una/s forma/s de enunciación oral espontánea, "accidentada”, con vacilaciones, son observadas como tensiones que tienen el potencial de construir o reforzar argumentos de exclusión, los cuales a menudo se fundamentan en criterios “objetivos" procedentes de la lingüística prescriptiva de inspiración escritural, tanto como en los pilares de gramaticalidad, uniformidad y riqueza expresiva en los que basa su autoridad para evaluar y censurar la lengua oral. Esta mirada glotopolítica parte de considerar que estos criterios objetivos, como los pilares de gramaticalidad, uniformidad y riqueza, coinciden con los usos lingüísticos de la burguesía y de las élites intelectuales y de gobierno. 
generales planteadas en la teoría de translanguaging (García 2009; Otheguy, García \& Reid 2015). Como se verá más adelante, esta perspectiva sobre los procesos cognitivos de hablantes bilingües puede aportar a la explicación de algunos fenómenos de vacilación identificados en nuestro corpus, en tanto que aparecen como marcas que reflejan momentos de un intenso auto-monitoreo por parte de los hablantes. ${ }^{3}$ Esos momentos de elevado auto-monitoreo en una situación interactiva formal podrían observarse como cognitivamente similares a los que experimentan los hablantes bilingües cuando eligen palabras del inglés o el español de su repertorio lingüístico para adaptarse socialmente a la situación en que se encuentran, como plantea la teoría de translanguaging. De forma similar, otros estudios (Clark 1994; Duez 2001), también enfatizan la función de planificación discursiva que aparentan tener determinados fenómenos de vacilación.

Cuando algunos fenómenos de vacilación parecen operar como elementos de apoyo en la planificación de los enunciados del discurso (y por lo tanto constituirse como una expresión oral que refleja el proceso mediante el cual se realiza esa planificación), también aparentan conllevar, por lo tanto, funciones de cohesión argumentativa. En otras palabras, los fenómenos de vacilación no solo contribuirían a la planificación de los mensajes del hablante, sino que también permitirían unir y relacionar semánticamente segmentos de información en el proceso de construcción del mensaje (Ávila 1999; Jeong 2011). Esta afirmación, no

3 En este caso el auto-monitoreo de hablantes en situaciones de habla formal o protocolar, como las que se dan al interior del Congreso de la Ciudad de México que es el ámbito institucional aquí estudiado. 
obstante, no pretende apoyar la idea de que la presencia de fenómenos de vacilación signifique necesariamente que acontece exclusivamente para realizar alguna de esas dos funciones o ambas (i.e. planificadora y cohesionadora), por el contrario, los datos que se presentan más adelante muestran que las vacilaciones pueden aparecer con otros valores o funciones comunicativas, o no tenerla, como es el caso de algunos sonidos vocálicos o consonánticos que se hacen presentes como fillers (ver Bortfeld et al. 2001; Clark \& Fox Tree 2002; Candea 2008; Watanabe et al. 2008 ), que en el contexto aquí analizado operarían como elementos fónicos que saturan el silencio de sonido.

Mostrar cómo algunos de estos fenómenos, en especial aquellos que parecen funcionar como planificadores discursivos, evidencian momentos en los que el hablante realiza la operación cognitiva de seleccionar de entre los recursos de su repertorio lingüístico en un proceso similar al de un hablante bilingüe (Otheguy et al. 2015), presentando determinados patrones sintácticos y semánticos, es el objetivo principal del análisis que sigue, realizado a partir de una muestra de enunciados extraídos del corpus, y de su segmentación según categorías tradicionales del análisis gramatical, así como de la tipología básica de palabras propuesta por Schachter \& Shopen (2007). Se busca ofrecer así explicaciones sobre la aparición de los seis fenómenos de vacilación que aquí se estudian (sonidos breves, repeticiones no intencionales, sonidos largos, alargamientos de palabra, palabras cortadas y frases cortadas), según se relacionan con el grado de planificación discursiva de los enunciados en los que aparecen.

Es importante destacar que tanto la elección de las herramientas teóricas, los datos empíricos obtenidos etnográficamente del registro de un 
tipo de interacción concreto, como las reflexiones resultado del análisis, no tienen como propósito postular predicciones o leyes sobre el uso lingüístico en lengua española, ni sobre el "comportamiento" de ningún fenómeno en particular, ni mucho menos sobre un conjunto de fenómenos cuya mayor similitud es el hecho de no presentar una expresión equivalente en lengua escrita. Esta aportación pretende ser una descripción de la competencia lingüística de los hablantes que sirva como punto de partida para una profundización posterior, así como evidenciar que estos objetos ofrecen información valiosa de sus prácticas lingüísticas y discursivas, como de las complejas relaciones que se dan entre lo lingüístico, lo cognitivo y lo social (cf. Allwood, Nivre \& Ahlsen 1990).

Como suele suceder en estudios de caso, es importante reconocer que uno de los mayores retos ha sido el problema de la representatividad de la muestra y la justificación de su uso para la investigación. Es necesario, por lo tanto, insistir en que a pesar de que los datos corresponden a un tipo de interacción específica, asimétrica, que acontece en una institución gubernamental de la Ciudad de México, durante el proceso de "entrevistas" realizadas en el 2013 por un grupo de legisladores en la Asamblea Legislativa ${ }^{4}$ a ciudadanos que compiten por un cargo público, estas particularidades no les restan validez a los hallazgos, en tanto que, como se ha dicho, no se pretende comprender el objeto de estudio a partir de

${ }^{4}$ Se trató de un proceso diseñado para seleccionar, de entre un conjunto de aspirantes, a aquel que los legisladores consideraron más "apto" o "apta” para el cargo de presidente honorario (ombudsperson) de la Comisión de Derechos Humanos del Distrito Federal (CDH) (ver $\$ 2.1$ infra). 
criterios positivistas, sino observar los hallazgos como parte de los resultados de una investigación etnográfica en un contexto de habla situada.

En la siguiente sección presentamos una descripción del entorno institucional del que se ha obtenido el corpus y los ejemplos con los que aquí se trabaja, así como el contexto interaccional. Se acota y justifica el espacio en el que toma lugar la problematización sobre las vacilaciones orales: la Asamblea Legislativa del Distrito Federal (hoy Asamblea Legislativa de la Ciudad de México), así como la descripción del evento discursivo específico, que corresponde al proceso de selección de la ombudsperson de la capital mexicana, proceso cuya responsabilidad corre a cargo de los legisladores adscritos a esa Asamblea Legislativa.

\section{La Asamblea Legislativa del Distrito Federal:}

\section{CONSTITUCIÓN DEL CORPUS}

Este trabajo reporta resultados de un estudio que observó discursos que aparecen en un entorno político en particular, y, al interior de este, un tipo de encuentro específico. Ese entorno fue la vi Legislatura de la Asamblea Legislativa del Distrito Federal (Ciudad de México), en adelante ALDF o Asamblea, para el periodo que va de septiembre de 2012 a septiembre de 2015. Se escogió esta institución debido a que la investigación del discurso parlamentario mexicano, estudiado en el ámbito federal por Carbó (1987) y Vázquez Laslop (2011), no había sido explorado en el terreno local, ni en el tipo de interacción concreto aquí tratado al interior de estos. Además, en virtud de que en un principio el diseño de 
la investigación original persiguió un interés por explicar la variación de las vacilaciones de acuerdo con el sexo de los hablantes, se eligió a la ALDF pues saltó a la vista que del total de 66 curules 44 estuvieron ocupadas por hombres y 22 por mujeres. ${ }^{5}$

Como es común en el ámbito parlamentario mexicano, el trabajo de la ALDF se realiza a partir de la división de comisiones de trabajo, cada una especializada y dedicada a distintos temas relacionados con atribuciones y agendas de trabajo propias. Estas comisiones agrupan a los legisladores de la Asamblea de acuerdo con los intereses políticos de los grupos parlamentarios (distribución de la representatividad según los partidos políticos y sus posturas ideológicas), así como la biografía y especialidad profesional de cada legislador. Se determinó construir un corpus basado en el trabajo de estas comisiones debido a la importancia que representan al interior del trabajo legislativo. Además de configurar una diversidad de momentos de importancia para la toma de decisiones en el poder legislativo, las interacciones que se dan al interior de estas comisiones configuran usos lingüísticos y discursivos que no han sido abordadas por las ciencias del lenguaje.

Estas motivaciones, y en especial la preocupación original por llevar a cabo un estudio sociolingüístico de las vacilaciones con perspectiva de género, ${ }^{6}$ dieron lugar a una examinación de las diversas comisiones de la ALDF en términos del balance entre legisladores hombres y mujeres. El tra-

5 Uno de los intereses centrales que dieron origen a esta investigación fue la inequidad de género que persiste en la distribución y ocupación de cargos públicos en instituciones del gobierno mexicano.

6 El presente trabajo pretende ofrecer un suelo empírico que pueda servir de base para estudios posteriores, de modo que se centra la atención en los entornos léxicos y sintácticos de 
bajo de la Asamblea para el año 2013 estuvo organizado por 37 comisiones legislativas ordinarias, 9 comisiones especiales, 9 comités, y una Comisión Jurisdiccional. Entre sus funciones se encuentran dictaminar, atender y resolver iniciativas, proyectos y propuestas de leyes que, de ser aprobadas, son sometidas a votación en el Pleno de la Asamblea (Ley Orgánica 2008). Del conjunto de comisiones y comités, solo dos contaban con mayoría de hablantes mujeres: la Comisión para la Igualdad de Género, compuesta en su totalidad por mujeres ( $\mathrm{N}=9$ ), y la Comisión de Derechos Humanos, que contó con cinco legisladoras (una de ellas presidenta y otra vicepresidenta), y cuatro legisladores hombres (uno de ellos secretario). Fue por este "balance" que se determinó trabajar con esta última.

Además de dictaminar, atender y resolver iniciativas, proyectos y propuestas de ley, esta comisión legislativa (en adelante CLDH), tuvo como parte de sus atribuciones realizar la selección del presidente honorario (ombudsperson) de la Comisión de Derechos Humanos del Distrito $\mathrm{Federal}^{7}$ (en adelante $\mathrm{CDH}$ ). Para llevar esto a cabo, los legisladores de la CLDH diseñaron un trámite que dio inicio con la publicación de una convocatoria dirigida a la ciudadanía en general, y que culminó con la realización de 29 entrevistas a los aspirantes que aplicaron al cargo, rea-

\footnotetext{
las vacilaciones, quedando pendiente el reporte y la interpretación de otros hallazgos del corpus que integren una perspectiva de género.

7 "Organismo público autónomo con personalidad jurídica y patrimonio propios que tiene por objeto la protección, defensa, vigilancia, promoción, estudio, educación y difusión de los derechos humanos, establecidos en el orden jurídico mexicano y en los instrumentos internacionales de derechos humanos" (Ley de la Comisión de Derechos Humanos del Distrito Federal Cap. 1, Art. 2.)
} 
lizadas durante los días 29, 30 y 31 de octubre de 2013. De entre las 29 personas "entrevistadas", los legisladores eligieron a la ombudsperson de la ciudad. La coyuntura y la relevancia política y social de este evento motivaron su elección para conformar el corpus de la investigación.

\subsection{Corpus: las entrevistas para elegir a la ombudsperson}

El corpus se compone por 9 de esas 29 entrevistas realizadas por los legisladores de la CLDH para la selección de la ombudsperson para dirigir la Comisión de Derechos Humanos ( $\mathrm{CDH}$ ) de la ciudad. Cinco de los aspirantes fueron hombres y cuatro mujeres, conjunto que fue elegido de forma aleatoria de entre todas. En la Tabla 1 se presentan en orden cronológico los nueve aspirantes entrevistados, la duración de cada sesión y la fecha de realización.

Tabla 1. Composición del corpus

\begin{tabular}{cccc}
\hline $\begin{array}{c}\text { Número } \\
\text { de entrevista }\end{array}$ & $\begin{array}{c}\text { Aspirante } \\
\text { entrevistado }^{\mathrm{b}}\end{array}$ & $\begin{array}{c}\text { Duración } \\
\text { (minutos) }\end{array}$ & Fecha (2013) \\
\hline 1 & H1 & 59 & 29 de octubre \\
2 & H2 & 60 & 29 de octubre \\
6 & M1 & 58 & 29 de octubre \\
7 & H3 & 53 & 29 de octubre \\
10 & H4 & 47 & 29 de octubre \\
13 & M2 & 49 & 30 de octubre \\
16 & M3 & 41 & 30 de octubre \\
21 & H5 & 50 & 31 de octubre
\end{tabular}


Tabla 1. Composición del corpus (concluye)

\begin{tabular}{cccc}
\hline $\begin{array}{c}\text { Número } \\
\text { de entrevista }\end{array}$ & $\begin{array}{c}\text { Aspirante } \\
\text { entrevistado }\end{array}$ & $\begin{array}{c}\text { Duración } \\
\text { (minutos) }\end{array}$ & Fecha (2013) \\
\hline 24 & $\mathrm{M} 4$ & 45 & 31 de octubre \\
\hline
\end{tabular}

a El número de entrevista en la primera columna corresponde al orden dentro de la totalidad del evento (29 entrevistas), por esta razón los números no son consecutivos.

b La "H” y "M" en la segunda columna representan el sexo del entrevistado, mientras que el número corresponde al orden de aparición dentro de cada grupo, así H1 es el primer aspirante hombre y M1 es la primera aspirante mujer.

La duración total de estas nueve entrevistas fue de 462 minutos, esto es, 7 horas y 42 minutos de videograbación. El formato, es decir, las reglas de la interacción diseñadas y aprobadas por los y las legisladores para la realización de las entrevistas a los aspirantes, se reproduce en la Tabla 2.

Como se aprecia en la Tabla 2, las entrevistas estuvieron conformadas por secuencias estrictamente delimitadas en duración temporal para los aspirantes entrevistados. La secuencia de presentación de propuesta (2) fue de 15 minutos, la de respuestas a las preguntas de los legisladores (4) de 15 minutos y el cierre (5) de 5 minutos. La propia naturaleza enunciativa de cada secuencia, así como el alto grado de formalidad del evento comunicativo, constituyen en sí entornos condicionantes que explican tanto la diversidad como la variación de fenómenos lingüísticos y discursivos observados en cada una de ellas, entre estos, los distintos tipos de vacilaciones y sus frecuencias de aparición de una secuencia discursiva a otra.

Las seis secuencias de la Tabla 2 pueden ser observadas según el grado de planificación discursiva que presentan. Bajo esta óptica, la sección más planificada de todas sería la lectura del currículum (1) para introducir al o a la 
Tabla 2. Formato aprobado para las entrevistas a aspirantes a la $\mathrm{CDH}$

\begin{tabular}{|c|c|c|c|}
\hline & Secuencia & Tiempo permitido & Hablante \\
\hline 1 & $\begin{array}{l}\text { Bienvenida y presentación del } \\
\text { aspirante. } \\
\text { Lectura de su currículum vítae. }\end{array}$ & $\begin{array}{l}\text { Variable. (Entre } 3 \text { y } 5 \\
\text { minutos). }\end{array}$ & Diputada presidenta \\
\hline 2 & $\begin{array}{l}\text { Presentación de propuesta de } \\
\text { trabajo. }\end{array}$ & 15 minutos & Aspirante entrevistado \\
\hline 3 & $\begin{array}{l}\text { Formulación de preguntas al } \\
\text { aspirante. }\end{array}$ & $\begin{array}{l}\text { Variable. } 3 \text { minutos por } \\
\text { cada diputado aproxima- } \\
\text { damente. }\end{array}$ & $\begin{array}{l}\text { Entrevistadores (dipu- } \\
\text { tadas y diputados pre- } \\
\text { sentes) }\end{array}$ \\
\hline 4 & $\begin{array}{l}\text { Respuestas a preguntas de } \\
\text { diputados. }\end{array}$ & 15 minutos & Aspirante entrevistado \\
\hline 5 & Cierre. & 5 minutos & Aspirante entrevistado \\
\hline 6 & $\begin{array}{l}\text { Agradecimiento y despedida } \\
\text { al aspirante entrevistado. }\end{array}$ & $\begin{array}{l}\text { Variable. (Menor a } 1 \\
\text { minuto). }\end{array}$ & Diputada presidenta \\
\hline
\end{tabular}

aspirante, lectura que corrió a cargo de la diputada presidenta de la Comisión; se considera altamente planificada pues se trata de un documento escrito, resultado de la "representación editorial" de una vida profesional. Tanto la presentación (2) como el cierre (5) a cargo de los aspirantes también se consideran secuencias altamente planificadas; estos momentos de discurso expositivo buscan conseguir la simpatía o adherencia de los entrevistadores, los legisladores, y constituyen el despliegue discursivo mediante el cual compiten por el cargo y, por lo tanto, muestran características de una argumentación destinada a persuadirlos (cf. Perelman \& Olbrechts-Tyteca 2010). Esta intención retórica es compartida por las presentaciones y cierres de todos los aspirantes en todas las entrevistas, y concuerda con otros 
rasgos discursivos que también aparecen en esas secuencias, como por ejemplo el hecho de que muestran un orden o estructura cuidados, énfasis de determinados temas, así como prestan gran atención a la articulación y a la entonación de los enunciados. Es posible que algunas de estas intervenciones hubiesen sido ensayadas o incluso memorizadas. A estos supuestos que apuntan hacia una planificación anticipada de tales secuencias, se suma la evidencia de que presentan un grado mucho menor de aparición de fenómenos de vacilación, como se verá más adelante.

En el extremo opuesto de planificación se encuentra la secuencia que corresponde a respuestas (4) a las preguntas de los legisladores. Se toman estas respuestas (4) como el momento de menor planificación de las entrevistas dado que los aspirantes respondieron a preguntas para las que no contaban con una respuesta preparada con anticipación, como sí fue el caso de la presentación (2) y el cierre (5). Para los propósitos del análisis lingüístico que se plantea en este trabajo resulta interesante comparar la secuencia (2), presentación, con (4), respuestas, dado que como se puede observar en la Tabla 2, ambas tuvieron aproximadamente la misma duración temporal y permiten por lo tanto el contraste de dos momentos discursivos distintos en los que participan los mismos hablantes con la misma asignación de tiempo. En términos del grado de planificación (2) este contraste constituye el momento de mayor planificación y (4) el menor, aunque ambos conservan su carácter formal y protocolar impuesto por el evento comunicativo del que forman parte.

Dado que los 9 aspirantes atravesaron por el mismo proceso es posible identificar patrones discursivos que contribuyen a caracterizar determinado momento o secuencia dentro de la interacción. La secuencia de preguntas (3) se configura como un turno de habla colectivo en el que los 
diputados presentes (no todos los diputados de la CLDH participaron en todas las entrevistas del proceso de elección de la ombudsperson) plantearon sus preguntas a los aspirantes, en algunos casos se trató de preguntas que habían sido planificadas con antelación y repetidas de forma sistemática de un aspirante al otro, mientras que en otros casos fueron motivadas por las presentaciones o la lectura del currículum. Estos enunciados han sido identificados como planificados o "semi-planificados", pero en ningún caso enteramente no planificados, ya que incluso en su forma más espontánea, su formulación por parte de los legisladores puede estar aprovechando el tiempo que les da (1), lectura de currículum, y (2), presentación del aspirante, para la elaboración de sus preguntas.

Los aspirantes se presentaron ante la CLDH con una propuesta de trabajo (2) que prepararon con anticipación; a partir de esa exposición los diputados realizaron preguntas, a las que los aspirantes tuvieron que dar respuesta sin un plan discursivo previo. En esta secuencia de respuestas (4), por tanto, puede observarse una argumentación mucho más espontánea, con un orden y desarrollo de ideas más libre y, efectivamente, con una mayor presencia de fenómenos de vacilación. Como se verá, es en esta sección de respuestas donde proporcionalmente aparece la mayor cantidad de ocurrencias, y es este el motivo por el que el presente análisis se centra en datos extraídos exclusivamente de esta sección.

\subsection{Las vacilaciones en la secuencia de respuestas}

Las nueve entrevistas presentadas en la Tabla 1 fueron transcritas ortográficamente, de manera similar al método que emplea Fant (1996) para transcribir usando "palabras gráficas", es decir, separadas por espacios, 
y posteriormente fueron etiquetadas siguiendo las convenciones del Proyecto para el Estudio Sociolingüístico del Español de España y América, en línea (pRESEEA 2008). A la hora de llevar a cabo el proceso de transcripción, se enfrentó la inevitable tarea de determinar lo que en el discurso contaba como vacilación y lo que no, razón por la que en la siguiente sección se ofrece una definición operativa de la categoría para ser aplicada en la identificación de los fenómenos en las transcripciones.

Es importante señalar que para la transcripción sociolingüística, PRESEEA cuenta con la etiqueta <vacilación/>, la cual se utiliza para marcar casos de "titubeo breve", descripción que, a nuestro parecer, no agota la riqueza de posibilidades orales que son objeto de interés en este estudio. Por esa razón, se crearon las siguientes formas de etiquetado:

\begin{tabular}{ll} 
Tabla 3. Etiquetado de fenómenos de vacilación \\
\hline Fenómeno & Etiqueta \\
\hline Sonido breve & $<$ sonido $>$ \\
Repetición & $<$ repetición $>$ \\
Sonido largo & $<(:)>$ \\
Alargamiento de palabra & $<(:)>$ \\
Frase cortada & $<$ frase_cortada $>$ \\
Palabra cortada & $<$ palabra_cortada $>$ \\
\hline
\end{tabular}

En el corpus de 9 entrevistas se observó un total de 2575 casos de estos seis tipos de vacilación. La secuencia de respuestas (4) concentró el $41.8 \%$ de ese total, es decir 1077 ocurrencias, un promedio de 120 por cada aspirante en el lapso de 15 minutos que duró la secuencia. Estas 
cifras contrastan con la presentación (2), donde apareció un total de 470, es decir, 52 por cada entrevistado en promedio, y con una duración de 15 minutos también. Este incremento de más del doble es justamente una evidencia de la estrecha relación entre el grado de planificación discursiva y el número de vacilaciones en el discurso, al menos en este contexto comunicativo.

\section{VACILACiONES, CLASES DE PALABRAS, PLANIFICACIÓN DisCURSIVA Y TRANSLANGUAGING}

Tanto el material recolectado como la trayectoria del análisis seguido, plantean un estudio sociolingüístico consistente en aislar fenómenos lingüísticos específicos — los fenómenos de vacilación - para intentar explicarlos a la luz de sus formas léxicas y sus relaciones sintácticas según el modelo de Schachter \& Shopen (2007), así como su relación con los aspectos cognitivos de la producción del lenguaje (languaging) establecidos desde la teoría de translanguaging (García 2009; Otheguy, García \& Reid 2015). Antes de pasar al análisis de los datos, se hace necesario precisar algunos conceptos sobre los que se trabaja.

\section{1 ¿Qué son las vacilaciones?}

Como se ha señalado en otras oportunidades (Quesada 2019), es necesario reconocer que plantear una definición teórica que consiga explicar y abarcar todas las posibilidades de la categoría "vacilación” resulta proble- 
mático pues las expresiones de este objeto de estudio presentan una gran variación según las situaciones sociales o "registros”, según los fenómenos particulares que se estudian, según la manera en que estos se agrupan o las funciones discursivas, comunicativas/conversacionales, lingüísticas, o cognitivas que pueden presentar. Cribble (2018), quien ha problematizado la categoría de vacilación a partir de la idea de fluidez oral en inglés y francés, se refiere a la tipología de fenómenos de vacilación que estudia $(\mathrm{N}=10)$, como "fluencemes", vocablo que adopta de Götz (2013). Su propuesta parte de establecer que estos fluencemas no representan por sí mismos un problema de comunicación, al margen de si son apreciados como una categoría general o como fenómenos específicos:

[fluencemes] are not necessarily problematic but rather reflect some cooperative (even listener-oriented) search for the optimal utterance. Fluency is the result of these signaling strategies: it does not equate to absence of fluencemes, but rather efficient (sometimes even creative) use of them, where efficiency is defined inter-subjectively and in context by the co-participants. Disfluency, on the other hand, corresponds to the perceptive effect of more symptomatic uses of fluencemes, through which the speaker is expressing some sort of production trouble, leading to major disruptions in the prosody and/or syntax. (Götz 2013: 22).

Esta definición, como se observa, reconoce y enfatiza el carácter situado de la interacción y de la intersubjetividad en las percepciones sobre la fluidez ("eficiencia") o la "disfluidez" que resultan de gran valor. De manera complementaria a la aportación de Cribble, el acercamiento 
etnográfico al evento comunicativo específico realizado en esta investigación, la revisión de trabajos teóricos y empíricos sobre distintos tipos de vacilación y la observación de las entrevistas en la CLDH, su videograbación y transcripción, son elementos que también ofrecen bases para elaborar una definición operativa de lo que cuenta como "vacilación” en este estudio. Una vacilación es por lo tanto entendida como aquella unidad de la expresión oral que se manifiesta en forma de "interrupción” o ruptura léxica, sintáctica o prosódica, producida por el hablante, sobre el enunciado o enunciados que conforma o conforman su propio turno de habla; esta ruptura puede aparecer en forma de pausa de silencio o pausa de sonido, que puede ser breve o larga, encontrarse aislada como elemento léxico (casos como el pronombre demostrativo este, común en español) o como elemento no léxico (como el sonido $e$, corto o largo, también común en español), o como la prolongación vocálica o consonántica de una palabra (i.e.: este $<(:)>)$, y en forma de repetición no intencional de algún elemento léxico o no léxico. Esta definición excluye casos de marcadores discursivos (que por lo común aparecen al inicio del turno de habla, o en momentos que apoyan la formulación inicial de enunciados), interjecciones repentinas, hipo, risa, estornudos, bostezos y cualquier otro sonido del propio hablante provocado por factores externos del ambiente.

Es importante resaltar que, aunque esta definición resulta satisfactoria en la medida en que permite dar un contorno a los fenómenos que nos interesa estudiar, es cierto que conlleva cierto prescriptivismo, en el sentido de que acude a una noción de lengua fluida y continua, al apuntar a elementos que "interrumpen” el enunciado, y en el sentido de que 
esa noción de lengua reproduce un estándar de lengua oral establecido a partir de la lengua escrita. Esta definición parece asumir, por lo tanto, que la lengua oral y sus rasgos configuran una especie de "desviación" a la lengua escrita que debe ser entendida y explicada, como si se tratara de un registro "marcado", y en la que el estado "normal" del uso se inspira en expectativas de "fluidez", "continuidad" y no interrupción. En otras palabras, uno de los efectos colaterales de la definición propuesta es el hecho de que permite una objetivización/aislación de estos rasgos orales para su observación y descripción, que trae nuevos retos a la investigación, ya que al hablar de "rupturas" léxicas, sintácticas o prosódicas producidas por el hablante sobre el enunciado o enunciados al interior de su propio turno de habla, inevitablemente se replica la idea de unidad y continuidad del discurso, y de las "rupturas" como un fenómeno excepcional que debe ser cuestionado y analizado por las ciencias del lenguaje. ${ }^{8}$ Aunque la definición de "vacilación" nos permite avanzar, conviene tener presente que no se trata de una categoría absoluta, y que su uso acarrea una serie de consecuencias que deben ser al menos reconocidas.

Resulta relevante referir el trabajo de Allwood et al. (1990) pues su propuesta no solo reconoce que la dicotomía langue-parole al interior de la ciencia lingüística ha sido empleada como excusa para excluir fenómenos orales del "estudio serio", y de tal forma, preservar de manera indirecta lo que de acuerdo con los autores se ha llamado the written language

8 Consideramos que una profundización sobre cómo se configuran los trámites, los acuerdos o los procesos mediante los que se construye el saber lingüístico resulta de gran importancia, pues de ellos depende lo que se considera "excepcional", "marcado" o de relevancia para llevar a cabo una investigación. 
bias. El trabajo de Allwood et al. (1990) constituye una rica aportación al campo de estudio de estos fenómenos, los cuales refieren como speech management phenomena (SM). En su propuesta, los SM involucran un comportamiento lingüístico que evidencia que un hablante gestiona su comunicación al mismo tiempo que toma en cuenta a su interlocutor, produciendo miradas, pausas, gestos, repeticiones, cambios en el contenido o expresiones ya formuladas, etcétera. Para estos autores la estructura y funciones de la lengua oral y escrita están relacionados con al menos seis factores: el entorno físico de la comunicación, el entorno cultural, los participantes en la conversación y sus características (biológicas, psicológicas, sociales), la naturaleza de las actividades que persiguen los participantes, la interacción comunicativa entre estos en tanto se desarrolla según objetivos y actividades comunes y el contenido informacional o tema de las contribuciones (cf. 1990: 2).

De acuerdo con Allwood et al. (1990), el estudio de los fenómenos de SM no debería partir de una perspectiva psicolingüística intraindividual ni desde una perspectiva exclusivamente social, "on our view, at least both of theses perspectives should be investigated simultaneously" (1990: 8). En su propuesta de análisis, clasifican los fenómenos de SM según su estructura y función, que dividen en dos tipos principales: (i) choice related sM-functions y (ii) change related sM-functions. Al respecto establecen que:

The function of choice related SM is to enable the speaker to gain time for processes having to do with the continuing choice of content and types of structured expression. More in particular, such processes can be connected 
with prompting memory, search of memory, hesitation and planning. (Allwood et al. 1990: 10).

Mientras que en los fenómenos de change related sM su función es:

$[\ldots]$ to enable the speaker on the basis of various feedback processes (internal and external) to change already produced content structure or expressions. The subset of these processes that are based on the speaker's reaction to his own speech production make up the kernel of what in Allwood (1986) was called IFB -intraindividual (communicatively related) feedback. When change related SM is normatively motivated, it can be characterized as selfrepair (Schegloff 1978) or self-correction (Levelt 1983). (Allwood et al. 1990: 10).

Consideramos que estas aportaciones de Allwood et al. (1990) dialogan con el enfoque y el análisis de los datos de este trabajo, y ofrecen una definición de estos fenómenos que enriquece e informa la aquí planteada:

A common element of all SM processes is that they [...] require time. This means that they all can be used to gain time [...]. Two other common elements of all SM processes [...] are that they, on the one hand, require a temporary break in continuous speech production and, on the other hand, require mechanisms for normal speech production to be resumed. We therefore distinguish break and resumption as two SM functions which only exceptionally occur independently. (Allwood et al. 1990: 11). 
Estas cualidades de los SM descritas por Allwood et al. (1990), como se verá más adelante, aplican de manera particular para el caso de las palabras y frases cortadas.

\subsection{Tipos de palabras}

Para el análisis lingüístico, se recurre a algunos conceptos teóricos elaborados desde la tipología sintáctica. Algunas de las herramientas propuestas por Schachter \& Shopen (2007) resultan de utilidad para cumplir los objetivos de este estudio, adoptando una perspectiva que cuestiona el análisis gramatical que emplea categorías aplicadas a la lengua escrita. Para esto, Schachter \& Shopen citan al lingüista danés Otto Jespersen, cuando este asegura que:

I am firmly convinced many of the shortcomings of current grammatical theory are due to the fact that grammar has been chiefly studied in connection with ancient languages known only through the medium of writing, and that a correct apprehension of the essential nature of language can only be obtained when the study is based in the first place on direct observation of living speech and only secondarily on written and printed documents. (Jespersen 1924: 7).

A partir de esa idea, Schachter \& Shopen (2007) problematizan las categorías de la gramática tradicional y plantean que la clasificación de las partes del habla debe tomar en cuenta 1) la distribución de la palabra en la oración; 2) su rango de función sintáctica; y 3) sus categorías sintácticas 
o morfológicas; según estos criterios, los autores establecen que todas las lenguas del mundo tienen únicamente dos clases de palabras claramente identificables: palabras de clase "abierta” y palabras de clase "cerrada”. Las abiertas corresponden a léxico cuyo significado puede variar en el tiempo, de un hablante a otro, o de un dialecto a otro, son unidades que llevan carga semántica, sujeta al cambio y a la variación: corresponderían a sustantivos, verbos, adjetivos y adverbios, principalmente. Las cerradas, por el contrario, son menos susceptibles de modificar su significado en el tiempo y tampoco muestran gran variación de un hablante a otro, o de un dialecto a otro, este sería el caso de conjunciones, pronombres, clasificadores, cuantificadores, clíticos, entre otros. De acuerdo con Schachter \& Shopen (2007: 23), las palabras de la clase cerrada tienden a jugar un rol mucho más importante en lenguas analíticas, lenguas sin derivación morfológica que dependen del orden de las palabras para comunicar significados, mientras que tenderán a ser menos empleadas en lenguas de tipo sintético, lenguas cuyas palabras presentan derivación morfológica en algún grado y en las que las relaciones sintácticas o gramaticales entre sus elementos no dependen del orden en que se presenten, puesto que pueden aparecer codificadas en las declinaciones de su morfología.

\subsection{Elaboración y planificación discursiva}

Por elaboración discursiva oral se entiende el proceso que involucra elegir de entre los recursos léxicos, prosódicos, sintácticos y semióticos en general (así como de los elementos paralingüísticos que lo acompañan en la complementación de significados: tono de la voz, velocidad de elocución, 
expresión facial, gesticulación, entre otros), aquellos que el hablante evalúe adecuados para construir su mensaje según la interacción o situación comunicativa en que se encuentre. Esta elaboración discursiva puede ser 1) espontánea (no planificada), en la que el proceso de planear/pensar y el acto de hablar son simultáneos, o 2) planificada, donde el hablante ha preparado su discurso con anticipación a la situación interactiva. En este segundo tipo de elaboración discursiva el hablante ha contado con tiempo previo, lo que crea un desfase o distancia entre el proceso de planear/pensar y el acto de hablar.

Las expresiones de la lengua escrita, como las de la lengua oral presentan grados de planificación y espontaneidad que han sido integrados en algunos modelos para el análisis de la lengua (Koch \& Oesterreicher 2007 [1990]; Briz 2001). Se hace necesario tomar en cuenta que por muy espontáneo que pueda llegar a ser el acto de escribir, incluso escribir en un teclado de computadora, requiere o demanda más tiempo que el acto de hablar; por lo común, quien escribe cuenta con mayores oportunidades para planificar su mensaje, estas oportunidades aparecen y son tomadas de manera previa a la materialización gráfica del mensaje. La lengua escrita ofrece oportunidades para el ajuste, edición o "mejoramiento" del enunciado antes de que sea entregado al destinatario, oportunidades de ajuste, edición y "mejoramiento" que operan de manera distinta en los mensajes orales.

Paralelamente, por altamente planificada que sea la lengua oral, no deja de ser una práctica en la que hablar y pensar suceden casi de forma simultánea, y en la que el hablante realiza un auto-monitoreo del discurso (cf. Levelt 1983) en el que las condiciones externas de uso de la len- 
gua, es decir el contexto social, situacional o interpersonal, motivarían un mayor o menor "proceso editorial" cuya "evidencia material" serían fenómenos como las rupturas sintácticas, las vacilaciones, los sonidos, las repeticiones no intencionales, los refraseos, las reparaciones y reformulaciones, fenómenos que en conjunto pueden ser referidos como marcas de una "escasa destreza oratoria", según han sido referidos por el Instituto Cervantes (2008: 113), atentando contra la fluidez como ideal de la oralidad y contra la estética verbal promovida por la tradición retórica occidental y sus expectativas de unidad y continuidad discursiva.

\subsection{Translanguaging, auto-monitoreo y recursos semióticos}

Como ya se ha dicho, el presente texto es resultado de una investigación de corte etnográfico que se enfoca y busca contribuir a la comprensión de fenómenos que ocurren en el habla de las personas, en el nivel de sus competencias o prácticas discursivas, en una interacción situada, y no tanto en elaborar generalizaciones que sean aplicadas en el nivel del "comportamiento de la lengua”. Esta meta es la que nos posibilita hacer uso de algunos supuestos analíticos planteados desde la teoría de translanguaging, propuesta por García (2009) en sus estudios sobre educación bilingüe en la ciudad de Nueva York, así como por Otheguy, García $\&$ Reid (2015). Estas aportaciones podrían identificarse como parte de un campo disciplinar ajeno por el tipo concreto de hablantes y por sus objetivos de investigación (la comprensión de las prácticas bilingües), sin embargo, resultan valiosas y relevantes para el presente estudio por el procedimiento analítico que realizan y que es justamente el que aquí se 
retoma, y que lo mismo aplica para hablantes bilingües como monolingües, ${ }^{9}$ como se muestra en el resto de esta sección.

Para estos autores, la perspectiva de translanguaging parte de reconocer la premisa de que las lenguas, o lo que socialmente ha sido aceptado y reconocido como "lengua", es decir entidades como el "francés", el "inglés", el "aimara”, el "español”, son más un objeto social que un objeto lingüístico en sentido estricto. Establecen que lo que sí constituye un "objeto lingüístico" es lo que denominan "idiolecto" de un individuo particular, el cual es definido por los rasgos léxicos y estructurales que pone en práctica al hablar, y no, por el contrario, las "lenguas con nombre", que son abstracciones culturales asociadas con, o atribuidas a, una nación o algún grupo social, y cuyos contornos no pueden definirse únicamente a partir de rasgos léxicos o gramaticales (cf. Otheguy et al. 2015: 281).

Tomando esta idea como punto de partida, Otheguy et al. (2015) plantean que la sociolingüística tradicional, al buscar definir o entender las prácticas de hablantes bilingües, ha seguido el paradigma en el que las dos lenguas existen, $y$, al hacerlo, define y entiende dichas prácticas desde una mirada externa y ajena a los hablantes. Considerando la propia perspectiva de los hablantes, aseguran los autores, podemos darnos cuenta de que esas dos "lenguas" en realidad operan como un único idiolecto o "repertorio" de recursos lingüísticos en la mente de los bilin-

9 Se hace necesario reconocer, no obstante, que la investigación no indagó sobre si los hablantes de los que se han extraído los datos de este reporte son bilingües o multilingües y en qué grado. Sin embargo, al tratarse de aspirantes con un alto nivel de escolaridad, como se puede observar en sus currículos, se asume que han adquirido y son competentes en una segunda lengua, muy seguramente el inglés. 
gües. Así, lo que comúnmente se identifica como una "mezcla" de "lenguas", constituye una práctica de lo que refieren como translanguaging:

The two named languages of the bilingual exist only in the outsider's view. From the insider's perspective of the speaker, there is only his or her full idiolect or repertoire, which belongs only to the speaker, not to any named language. Translanguaging is the deployment of a speaker's full linguistic repertoire without regard for watchful adherence to the socially and politically defined boundaries of named (and usually national and state) languages (Otheguy et al. 2015: 281). ${ }^{10}$

Así, los autores deconstruyen la definición de "lengua" proponiendo dos sentidos: (a) uno en el que la lengua es un objeto socio-políticamente "construido, mantenido, y regulado", y (b) una definición de lengua entendida como entidad sin nombre, como conjunto de recursos léxicos y estructurales que constituyen el repertorio de cada individuo y que son empleados para comunicar:

Sense (a) of language, the social sense, encompasses the countable and easily enumerated entities that are tightly associated with established peoples or nations [...]. Sense (b) of language, the mental or psychological sense, encompasses the billions of individual linguistic competences of speakers the world over, irrespective of whether we call them monolingual or multilingual (Otheguy et al. 2015: 286).

10 Itálicas en el original. 
Con esta distinción de la categoría "lengua", los autores retoman el término de ideolecto originalmente acuñado por Bloch (1950), que definen como:

[...] a person's own unique, personal language, the person's mental grammar that emerges in interaction with other speakers and enables the person's use of language. An idiolect is language in sense (b) above, language viewed from the internal perspective of the individual, language seen separately from the external perspective of the society that categorizes and classifies named national languages. Idiolects are structured lists of lexical and grammatical features, that is, they are lists subdivided in components (e.g. lexicon, phonology, morphosyntax) and subcomponents (words belonging to one noun class or another, systems of tenses, systems of case endings or pronouns, etc., and, in some theories, movement, feature checking, etc.). An idiolect, then, is the system that underlies what a person actually speaks, and it consists of ordered and categorized lexical and grammatical features. (Otheguy et al. 2015: 289).

Los autores establecen la especificidad de los idiolectos al asegurar que no existen dos que sean idénticos. El idiolecto constituye así un objeto lingüístico formado por elementos léxicos y estructuras que, en el caso de los hablantes bilingües de inglés y español, forman un repertorio semiótico que es más amplio que el de los hablantes monolingües. Lo que tradicionalmente se ha observado como una "mezcla" de palabras o rasgos gramaticales entre el inglés y el español en los hablantes bilingües, plantean Otheguy et al., por lo tanto, no es tal si tomamos en cuenta que el 
inglés y el español son lenguas en el sentido (a), es decir, no definidas mediante criterios lingüísticos propiamente, sino por criterios socio-políticos (Op. Cit.), y si consideramos además que los hablantes bilingües solo emplean los recursos con los que cuentan en su repertorio, sin que exista una clara distinción interna entre lo que constituye una palabra o rasgo gramatical propio de una lengua y lo que constituye una palabra o rasgo gramatical de la otra.

Pero al margen de la discusión sobre lo que se considera la "lengua" o "lenguas" de los hablantes bilingües, en el estudio de los autores destaca el hecho de que los hablantes bilingües de español e inglés realizan un auto-monitoreo de su forma de hablar que ocurre de modo "más intenso" en comparación con el auto-monitoreo que realizan los hablantes monolingües. Los bilingües se adaptarían al lugar o situación social realizando operaciones como "use jugo in Cuba but not in Spain; use zumo in Spain but not in Cuba; do not use juice in either Cuba or Spain" (Otheguy et al. 2015: 292). Así, los autores explican que al hablar elegimos los recursos lingüísticos que vamos valorando "apropiados" según el contexto espacial, social, o situacional en el que estamos, recursos que se encuentran en el repertorio de opciones semióticas de cada hablante y que, según esta propuesta, serían mayores para el caso de hablantes bilingües:

[...] the idiolects of monolinguals and bilinguals are not qualitatively different, only quantitatively different. The difference is that the idiolects of bilinguals contain more linguistic features and a more complex sociocultural marking of which features to use when and where. (Otheguy et al. 2015: 292). 
Este proceso de selección de recursos semióticos (sean léxicos o de cualquier otro tipo), por supuesto, constituye un comportamiento que se da en cualquier hablante, sea monolingüe o no, lo que aquí nos interesa destacar es el hecho de que para Otheguy et al. (2015) este proceso es cognitivamente más complejo ("more intense", v. infra) en hablantes con repertorios más amplios, como sería el caso de los hablantes bilingües o multilingües. $\mathrm{Al}$ respecto, los autores apuntan que:

[...] all speakers, even monolinguals, monitor their speech to some extent in order to adapt to the interlocutor and social situation at hand. Our point has been that since bilinguals have idiolects with larger sets of lexical and structural features than monolinguals, $[\ldots]$ their monitoring is more intense than is usually found in monolinguals. (Otheguy et al. 2015: 297).

Como se puede deducir de estos planteamientos, subyace en esta teoría un componente cognitivo en el acto de hablar, es decir, una operación de búsqueda, selección y supresión de recursos léxicos o estructuras, para adaptar el idiolecto a determinadas situaciones sociales según resulten apropiadas. Si bien la propuesta de Otheguy et al. (2015) se desarrolla en el campo de los estudios sobre bilingüismo (marco al interior del cual proponen el proceso de translanguaging, por el cual los hablantes emplean recursos lingüísticos según sus necesidades comunicativas, sin considerar si se respetan o no los límites de alguna lengua en su definición como entidad sociopolítica) resulta valiosa para el análisis que aquí se elabora por incorporar la idea del monitoreo, o auto-monitoreo, que como se verá más adelante, tiene una correlación directa con la ocurrencia de los fenómenos de vacilación. 


\section{ANÁlisis}

El análisis de los contextos léxicos y sintácticos de las vacilaciones que aparecen en la secuencia de respuestas (ver secuencia 4 en Tabla 2, supra) partió del modelo de clasificación de palabras propuesto por Schachter \& Shopen (2007), esbozado en $\$ 3.2$ del apartado anterior. El total de casos de vacilación en la secuencia de respuestas fue de 1077 . A partir de la definición ofrecida en $\$ 3.1$ y del etiquetado y transcripción realizados en una hoja de cálculo de Microsoft Excel, se identificaron los siguientes fenómenos, como se indica en la Tabla 4:

\begin{tabular}{lc} 
Tabla 4. Vacilaciones en la secuencia de Respuestas & ${ }^{11}$ \\
\hline Fenómeno & Núm. Ocurrencias \\
\hline Repetición & 290 \\
Sonido breve & 258 \\
Sonido largo & 178 \\
Alargamiento de palabra & 75 \\
Frase cortada & 201 \\
Palabra cortada & 75 \\
\hline TOTAL & 1077 \\
\hline
\end{tabular}

Del total de 1077 enunciados se tomó una muestra de 199, que fueron seleccionados de manera aleatoria tratando de alcanzar el 20\% del

11 El orden de los fenómenos en la tabla ha sido dispuesto para coincidir con el análisis que sigue y obedece a un agrupamiento según las funciones discursivas que presentan. 
total de fenómenos en la secuencia de respuestas, y procurando una distribución representativa de cada fenómeno. A continuación, se desglosan los ejemplos por fenómeno, usando la categorización de palabras de Schachter \& Shopen (2007), y terminología de la gramatical española tradicional.

\subsection{Repetición $(N=36)$}

Para el caso de las repeticiones se analizaron 36 enunciados con repeticiones no intencionales o aquellas que no conllevan una función enfática en el discurso; en 30 de estos el elemento repetido fue una palabra de la clase cerrada (determinantes y preposiciones en la mayoría de los casos, aunque también aparecieron conjunciones, pronombres de relativo y clíticos de objeto directo), mientras que solo en 6 casos hubo repetición de palabras de la clase abierta ( 3 adverbios, 2 verbos y 1 sustantivo). En cuanto al elemento que sigue a la repetición se identifica que en la mayoría de los casos (20 de 36) la palabra posterior al fenómeno de vacilación pertenece a la clase abierta, como se observa en los ejemplos (1) (2) y (3), ${ }^{12}$ donde se destaca en negritas el elemento repetido y la palabra de la clase abierta que le sigue. En menor proporción, el elemento posterior a la repetición constituye una palabra de clase cerrada (16 casos de 36), como se observa en (4), donde luego de la repetición de la preposición "para" aparece un pronombre posesivo. Estos ejemplos y los subsiguientes no

12 El símbolo (/) en las transcripciones representa una pausa con duración aproximada de un segundo o menos. Cuando la pausa es de dos segundos se marca con dos diagonales (//), y así consecutivamente. 
constituyen secuencias, corresponden a enunciados producidos por distintos hablantes en varios momentos de la secuencia de respuestas a las preguntas hechas por los legisladores.

(1) / me parece que e <sonido $>$ debe de haber un un < repetición > sentido distinto / [C279]

(2) / por eso me parece que / la Comisión de Derechos Humanos debe jugar un papel de de <repetición> prevención / [C299]

(3) / obviamente el el <repetición > tema de cómo / de dar mayor celeridad / atención / a las áreas de la Sedeur / de Gobierno / [C284]

(4) I y qué vamos a hacer con esos / expedientes / tenemos que resolverlos de inmediato y canalizarlos para / para < repetición > su a- < palabra_ cortada $>$ su atención e $<(:)>/$ adecuada / [C275]

4.2 Sonido breve $(N=22)$

Del total de 199 enunciados estudiados, 22 correspondieron a casos en los que aparecían sonidos breves, como los que aparecen en los ejemplos (5), (6) y (7):

(5) e <sonido > participé en la Comisión Civil de Acompañamiento / [...] [C233]

(6) I a mí me parece que e <sonido> habría que estar considerando varias cosas / [C200]

(7) / lo mismo ocurrió con escenarios e < sonido > / muy complicados / del Parque Reforma Social / [C239] 
(8) / me parece que no es viable que e $<$ sonido > se esté planteando un tema / tan profundo / [C214]

De los 22 casos, 13 enunciados mostraron la aparición del sonido breve /e/ antes de palabras de la clase abierta, como los casos (5) y (6), donde el sonido antecede a un verbo, y (7), donde aparece ante un adverbio, mientras que en 9 ocasiones ese mismo sonido breve apareció antes de palabras de la clase cerrada, como el caso de (8), donde aparece antes de un pronombre "se".

\subsection{Sonido largo $(N=38)$}

Respecto al sonido largo, se revisaron 38 enunciados. La mayoría de las vacilaciones apareció antes de una palabra de la clase abierta (20 de 38), como sucede con los sonidos breves y las repeticiones. Estos casos pueden observarse en los ejemplos (9), (10) y (11). Hay que hacer notar, no obstante, que en los 18 casos restantes en los que el sonido largo ocurrió antes de palabras de la clase cerrada, 9 de ellos correspondieron a la introducción de frases preposicionales, como aparece destacado en negritas en los ejemplos (12), (13) y (14).

(9) en relación a lo que me e $<$ (:)> pregunta la diputada Laura Ballesteros / [C222]

(10) / e <(:)> tuvimos que intervenir para que efectivamente pudiera haber una mesa de recepción / [C238] 
(11) / es e $<($ :) > bien sabido que / el derecho de la manifestación / el derecho de la protesta es un derecho constitucional está ahí plasmado como como <repetición $>$ algo que es irrebatible / [C195]

(12) / al relatar / cuál ha sido mi planteamiento / $\mathbf{e}<$ (:) $>$ / en esta Comisión / he señalado una serie de ejemplos en los cuales / como abogado / particular / he estado cerca de la gente / [C228]

(13) / en relación a la pregunta del diputado Jorge Zepeda sobre los tratados internacionales / y cuáles han / quedado fuera / hay por supuesto grupos vulnerables // e <(:)> de gran atención que habría que considerar // [C323]

(14) / habrá que estar haciendo participar a los sectores empresariales / a todas las áreas / e <(:)> para / poder estar respondiendo al tema de los tratados internacionales / [C346]

\subsection{Alargamientos de palabra $(N=31)$}

En el caso de los alargamientos de palabra, se presenta el mismo patrón que en los sonidos largos: en la mayoría de los ejemplos el alargamiento ocurre antes de un elemento de la clase abierta (sustantivos, verbos, adjetivos, principalmente) en 20 de 31, y, en menor medida, antes de un elemento de la clase cerrada (determinantes, pronombres y preposiciones), en 11 casos. Los ejemplos (15), (16) y (17) son ejemplos de alargamientos de palabra que aparecen antes de sustantivos o verbos:

(15) / en lo particular me parece que / es un tema de avance / contra los 90 días que / se establecía antes de una $<($ :)> / retención / indebida desde mi punto de vista / [C368] 
(16) / en este sentido el tema / de la $<$ (:) $>$ Supervía / que se $<($ : $)>$ plantea / por parte del diputado Cuauhtémoc Velasco $/ \mathrm{e}<($ : $)>$ es e $<$ sonido $>$ precisamente e $<$ sonido $>$ el de e $<$ sonido $>$ estar e $<$ sonido $>$ más enfocado en los temas / de la conciliación / [C446]

(17) / le $<$ (:) $>$ respondería a la diputada Bertha Alicia / Cardona e $<$ (:) $>$ el tema / de las marchas que me parece que es un tema de $<$ frase_cortada $>$ $<$ reparación> sentido por la población / [C186]

Independientemente del elemento que sigue al alargamiento de palabra, hay que resaltar que en todo el corpus el sonido que se alarga es generalmente de tipo vocálico, además que el tipo de palabra en el que recae dicho alargamiento es casi siempre un elemento de la clase cerrada, de hecho, de estos 31 casos, 28 corresponden a esta clase y solo 3 de ellos se realizan con un elemento léxico de la clase abierta, como se observa en los ejemplos (18), verbo, (19), adverbio y (20), verbo también:

(18) / que ha significado / trastornos / muy importantes / e $<($ : $)>$ para la población en general / que ha $<$ (:) $>$ impedido e $<($ : $)>$ poder $\mathrm{e}<$ sonido $>$ / llevar a cabo acciones de trabajo / y ha sido realmente un tema muy complicado / [C190]

(19) / he observado de que e $<$ (:) $>$ cada $<$ (:) $>$ / área de gobierno / puede ser Procuraduría de Justicia / Secretaría de Seguridad Pública / e <sonido $>$ Secretaría de Gobierno / Comisión de Derechos Humanos / tienen una visión distinta de los derechos humanos / [C254] 
(20) / e <sonido > una Comisión de Derechos Humanos que no sea / e <sonido $>$ autónoma / que no tenga $<$ (:) $>$ e $<$ sonido $>$ una $<($ : $)>$ participación activa de las organizaciones civiles / [C383]

El caso de (21) es particular debido a que el alargamiento recae en un pronombre demostrativo "este", que a nuestro parecer resulta problemático de ubicar en las categorías de palabra "abierta” o "cerrada”, dado que la función que parece llevar en el enunciado es la de marcador discursivo. Sin pretender profundizar en la discusión o definición de dicha categoría, este ejemplo no ha sido considerado para ninguna de las dos clases de palabras, y ha quedado etiquetado como $<\mathrm{MD}>$. En el mismo ejemplo (21) aparece posteriormente el elemento "bueno", que también ha quedado etiquetado como marcador discursivo con función de reformulación, aunque en este caso no aparece rodeado de algún fenómeno de vacilación.

(21) / es un poco difícil <frase_cortada $>$ este $<$ (:) $><$ MD $>/$ e $<$ sonido $>$ / $<$ reparación $>$ bueno $<$ MD $>$ creo / que tengo la madurez suficiente / creo que tengo / la experiencia suficiente / porque no basta con que tenga un gran conocimiento / de los derechos humanos / no es suficiente / hay que tener otro tipo de atributos / sobre todo / la capacidad / en la toma de decisiones / [C1030]

4.5 Frases cortadas $(N=37)$

En cuanto a las frases cortadas, se revierte la tendencia de que la mayoría de los fenómenos de vacilación hasta ahora revisados (sonidos bre- 
ves, repeticiones, sonidos largos y alargamientos de palabra) aparezcan antes de palabras de la clase abierta, es decir, los cortes de frase, que en el corpus son realizados por lo común mediante una pausa de silencio, una repetición o un sonido breve o alargado, aparecen justo antes de un elemento de la clase cerrada, como se observa en los ejemplos (22), (23), (24) y (25):

(22) $/$ e $<($ : $)>$ el el $<$ repetición $><$ frase_cortada $>$ / < reparación $>$ en relación a a $<$ repetición $>$ lo que me e $<($ :) $>$ pregunta la diputada Laura Ballesteros / e $<(:)>$ si me considero una persona autónoma / e $<(:)>$ por supuesto que sí / [C220]

(23) /// e <sonido > los grupos vulnerables / por supuesto que / están e <sonido $>$ e $<$ sonido $><$ repetición $>$ los e $<(:)>$ trabajadores no asalariados / los niños de la calle / e $<(:)>$ los e $<$ sonido $>$ e $<$ sonido $><$ repetición $><$ frase_cortada $><$ reparación $>$ las personas de la tercera edad / [C383]

(24) // e $<(:)>/$ sobre la // vinculación de las resoluciones de la Comisión / igual < frase_cortada $>$ reparación $>$ de igual forma / tenemos los mecanismos / para hacer / valer $<$ frase_cortada $>^{13}$ / < reparación $>$ para hacer cumplir / estas recomendaciones / [C720]

13 Resulta interesante que este enunciado puede estar presentando dos valores: o bien es un corte de frase efectuado con el fin de "reparar" lo que se ha dicho e introducir una estructura que el hablante podría estar considerando como "mejor" o "más apropiada”, "para hacer cumplir"; o bien podría ser vista como una enumeración de elementos, con valor de intensificación del mensaje "hacer valer y hacer cumplir las recomendaciones". 
(25) $/$ e $<(:)>$ o- obviamente el el $<$ repetición $>$ tema de de $<($ : $)><$ repetición $>$ de $<$ repetición $>$ cómo $<$ frase_cortada $><$ reparación $>$ de dar mayor celeridad / atención / a las áreas de la Sedeur / de Gobierno / [C288]

En total se analizaron 37 enunciados con frases cortadas, de los cuales, 25 aparecen antes de elementos de clase cerrada (principalmente ante preposiciones, determinantes y conjunciones), mientras los 12 casos restantes fueron elementos de la clase abierta (adverbios, sustantivos, adjetivos y verbos).

4.6 Palabras cortadas $(N=35)$

En los casos de palabras cortadas los momentos de corte recaen en palabras de la clase abierta en 32 de 35 casos revisados, como se aprecia en los ejemplos (26), (27) y (28):

(26) / en relación a las med- < palabra_cortada > <reparación> a las modificaciones de la figura del arraigo / por la detención de control inicial / [C359]

(27) / porque debemos re- < palabra_cortada > / < reparación > de decir que las organizaciones de la sociedad civil / no son la sociedad / y no muchas $<$ frase_cortada $>$ / reparación > no muchos de los ciudadanos / pertenecen / o se <frase_cortada / a alguna organización / [C796]

(28) / pero finalmente es una atribución del gobierno federal / por lo que respecta a est- <palabra_cortada > <reparación > a estas problemáticas / [C1006] 
No obstante, al observar el elemento inmediato posterior al corte, en 20 casos ese elemento constituye una palabra de la clase cerrada, como puede apreciarse en las preposiciones de los mismos ejemplos (26), (27) y (28).

\section{Discusión}

Como se ha presentado en la sección previa, cuatro de los seis tipos de vacilación aquí revisados (sonidos breves, repeticiones, sonidos largos y alargamientos de palabra) aparecen antes de palabras de la llamada clase abierta en la mayoría de los casos. En el caso de las palabras cortadas el corte se da en palabras de la clase abierta casi en su totalidad. Esto no sucede así con las frases cortadas, que en su mayoría siguen un patrón que privilegia los cortes antes de elementos de la clase cerrada.

Esta observación es importante en virtud de que permite intuir las relaciones existentes entre las vacilaciones y otros elementos que forman parte del mensaje del hablante. Si se parte del supuesto de que las vacilaciones contribuyen a hilvanar o unir segmentos discursivos, donde la intención es lograr un mensaje claro, significativo y relevante, apropiado para el contexto en el que su enunciación toma lugar, es posible concluir que las vacilaciones reflejan un impulso del hablante por argumentar y transmitir un enunciado apropiado o preciso, y por este motivo podrían aparecer mayoritariamente antes de palabras de la clase abierta, es decir, palabras con contenido o peso semántico. Parecería entonces que las vacilaciones ocurren con mayor frecuencia antes de elementos de la clase 
abierta precisamente porque reflejan momentos de enunciación espontánea que no cuenta con un plan discursivo previo, evidencian también el proceso lingüístico y cognitivo en el que pensar y hablar son actividades que se dan de manera simultánea, y donde los hablantes tendrían mayor preocupación por conseguir transmitir un mensaje "idóneo", anclado a una situación social altamente protocolar y formalizada en la que el tiempo permitido para hablar se encuentra predeterminado (15 minutos), una situación en la que, por la misma razón, los hablantes se esforzarían por evadir el riesgo de producir mensajes que puedan llevar la posibilidad de una interpretación múltiple o ambigua en la audiencia, que en este caso son los legisladores de la CLDH, quienes se han constituido como jueces de los aspirantes y su actuación discursiva.

A primera vista, sin embargo, y tomando en cuenta exclusivamente la sintaxis de los enunciados, parecería que las frases cortadas plantean una excepción al resto de los tipos de vacilaciones, pues el corte sucede antes de palabras de la clase cerrada. Ante esto se hace necesario observar más allá de la adyacencia de las palabras con dicho corte, es decir, al elemento o elementos posteriores. Por ejemplo, en (29), que ha sido extraído y adaptado de (23) en el apartado anterior, se observa que tras el corte sigue enseguida una palabra de la clase cerrada, pero esta ha sido incluida para introducir el sustantivo "personas":

(29) $l o s<$ frase_cortada > <reparación > las personas de la tercera edad [C333]

Las palabras de la clase cerrada por lo general establecen relaciones entre otros elementos de la clase abierta y esta cualidad debe ser tomada 
en cuenta para el caso de las frases cortadas, donde, efectivamente, los cortes aparecen antes de palabras cerradas, sin embargo, parecería que el “objeto" o el "motivo" del corte no es en sí esta clase de léxico, sino las unidades que introducen en el enunciado, es decir, los cortes de frase se realizan aparentemente para adaptar el enunciado de manera que pueda recibir un concepto o idea que se va a decir, y que corresponde a algún elemento de la clase abierta, con contenido semántico, como es el caso de "personas" en (29). El determinante "los" debe ser reparado o "corregido" en cuanto ha sido dicho, debido a que la frase nominal introducida por el hablante correspondía al género femenino "personas de la tercera edad". Ante el problema que representa el hecho de que se ha pronunciado "los" como determinante de un referente femenino, el hablante detecta el inminente caso de falta de concordancia de género que esto le plantea e interrumpe la frase con un corte para reiniciarla "desde cero", modificando el determinante para que resulte gramaticalmente adecuado. En casos así, las frases cortadas muestran que, en este contexto comunicativo, los hablantes prefieren realizar un corte con reparación antes que producir una frase que resulte agramatical, inapropiada o imprecisa, donde el elemento relacionado con estos "problemas” aún está por decirse, como se observa en (29), y que corresponde a algún ítem léxico de la clase abierta. En este sentido, el "comportamiento" de las frases cortadas en particular parece seguir lo que Allwood et al. (1990) han referido como "change related sM-functions", definidas en $\$ 3.1$.

Las vacilaciones presentan una relación directa de planificación enunciativa con palabras de la clase abierta (verbos, sustantivos, adjetivos y adverbios) que muestra patrones en su distribución en la sintaxis de los 
enunciados. Cuando el elemento que sigue a la vacilación, específicamente del tipo frase y palabra cortada, es de la clase cerrada (palabras "funcionales"), el fenómeno es empleado para introducir por lo regular frases verbales, nominales, adverbiales o adjetivales, es decir, léxico con peso semántico, y por lo tanto estos cortes sirven como apoyos en la construcción del mensaje del hablante.

Por otro lado, si aceptamos la premisa de que las respuestas a las preguntas que los legisladores hacen a los aspirantes a ombudsperson de la $\mathrm{CDH}$ de la Ciudad de México constituyen momentos de un alto grado de auto-monitoreo en el discurso de los hablantes, en los que se abre paso un registro de habla formal que está siendo objeto de gran vigilancia, estos momentos de auto-monitoreo accionarían un esfuerzo cognitivo en tres sentidos: 1) conforman un contexto de habla que pone gran atención a la manera en la que se habla, debido en parte al protocolo y la solemnidad impuestos por el ámbito institucional en el que toman lugar; 2) conforman también un registro de habla en el que "lo discursivo" se vuelve el recurso primordial mediante el cual los aspirantes al cargo compiten para obtener la aprobación de su audiencia, compuesta por los legisladores de la CLDH; y 3) configuran un discurso que debe cuidar simultáneamente la producción de 1) y 2), y, adicionalmente, abrirse camino de manera espontánea, en la medida en la que constituye un mensaje que no ha contado con tiempo de planificación, como sí es el caso de la secuencia de presentación y la secuencia de cierre que corresponden a las secuencias (2) y (5) en la Tabla 2, y que por la misma razón muestran una mucho menor cantidad de fenómenos de vacilación en términos proporcionales. 
En esta triple tensión es en la que queda de manifiesto una expresión del "idiolecto" de los hablantes, como lo han planteado Otheguy et al. (2015), en un proceso de búsqueda de recursos de léxico-semánticos, o semióticos, similar al que activa un hablante bilingüe en momentos de translanguaging. Se concluye la similitud de estos momentos de elección semiótica del repertorio de los hablantes debido a que ambos persiguen la misma meta: producir un mensaje que resulte gramatical, pragmáticamente apropiado y semánticamente preciso. Si bien los aspirantes a ombudsman que aquí se estudian son hablantes monolingües de español al menos a simple vista, el proceso analítico de translanguaging sirve para confirmar que al hablar, sobre todo al hablar de manera no planificada, se evidencia el proceso mediante el cual se eligen y se descartan determinados recursos lingüísticos en función de la situación social en la que se tome lugar, ya sea que esos recursos lingüísticos se encuentren asociados al constructo "lengua", en su sentido socio-político, como por ejemplo "inglés" o "español", o que se encuentren asociados a las alternativas léxicas con las que un hablante cuenta dentro de una misma lengua, como cuando se ha aprendido que en algunos contextos sociales es más apropiado decir zumo y en otros jugo, para retomar el ejemplo de Otheguy et al. (2015). En otras palabras, esta propuesta teórica implica también considerar la idea de que en términos lingüísticos y cognitivos juice y jugo o jugo y zumo son todos el mismo significado, y son la situación externa y el contexto comunicativo los que determinan la forma de su significante, poniendo en entredicho las propias categorías de "bilingüismo" o "monolingüismo" de los hablantes, pues para hablar, más que internalizar y poner en práctica una determinada "lengua” o dos, es nece- 
sario elegir de un repertorio de recursos semióticos de cualquier nivel y en cualquier lengua o registro (léxicos, sintácticos, semánticos, pragmáticos, discursivos, paralingüísticos), elección que depende del ajuste a las condiciones sociales, y que demandan tiempo y planificación.

Sin tener conocimiento sobre si los aspirantes que participaron en este proceso de elección de ombudsperson son monolingües, bilingües o multilingües, se asume aquí que su lengua principal, la de su escolarización y de uso cotidiano es el castellano. De tal modo, el auto-monitoreo que realizan en una secuencia cruzada con las tensiones antes descritas estaría ocasionando una constante búsqueda y/o supresión de entre las opciones léxicas que aparecen en el repertorio semiótico disponibles en el idiolecto de cada hablante en una situación como la que aquí se ha descrito.

En esos procesos de búsqueda y/o supresión de recursos del idiolecto en momentos de alto grado de auto-monitoreo, los diversos fenómenos de vacilación realizarían una función de apoyo para la planificación discursiva espontánea no planificada. La manera en la que la mayoría de estos fenómenos de vacilación se relaciona con los elementos léxicos de los que se rodea, según pertenezcan a la clase abierta o cerrada, tanto como la manera en la que se distribuyen sintácticamente en los enunciados, constituyen, en última instancia, una evidencia que contribuye a verificar tal afirmación. 


\section{Convenciones de transcripción en los ejemplos}

$\begin{array}{ll}/ & \text { Pausa de 1 segundo (aprox.) } \\ / / & \text { Pausa de 2 segundos (aprox.) } \\ <\text { sonido }> & \text { Sonido breve } \\ <\text { repetición }> & \text { Repetición } \\ <(\text { :) }> & \text { Sonido largo } \\ <(\text { :) }> & \text { Alargamiento de palabra } \\ <\text { frase_cortada }> & \text { Corte de frase } \\ <\text { palabra_cortada }> & \text { Corte de palabra } \\ <\text { reparación }> & \text { El material siguiente constituye una reparación del } \\ & \text { hablante } \\ <\text { MD }> & \text { Marcador discursivo }\end{array}$

\section{REFERENCIAS}

Allwood, J.; Nivre, J.; y Ahlsén, E. 1990. Speech Management on the Non-Written Life of Speech. Nordic Journal of Linguistics 13(1). 1-45. Ávila, Silvia. 1999. El análisis del diálogo desde la perspectiva de los estudios de la interacción verbal: los marcadores de vacilación en la entrevista radiofónica. México, D.F.: UnAM. (Tesis de licenciatura).

Bloch, Bernard. 1950. Studies in colloquial Japanese IV. Phonemics. Language 26. 86-125. DOI: 10.2307/410409.

Bortfeld, Heather; Leon, Siliva D.; Bloom, Jonathan E.; Shchober,

Michael F. \& Brennan, Susan E. 2001. Disfluency rates in conver- 
sation: Effects of age, relationship, topic, role, and gender. Language and Speech 44(2).123-147. DOI: 10.1177/00238309010440020101. Briz Gómez, Antonio. 2001 [1998]. El español coloquial en la conversación: esbozo de pragmagramática. Barcelona: Ariel Lingüística.

Cameron, Deborah. 2012 [1995]. Verbal hygiene. Nueva York: Routledge. Candea, Maria. 2008. Contribution à l'etude des pauses silencieuseset des phénom è nes dits "d'hésitation" en français oral spontané. Etude sur un corpus de récits en classe de français. Universidad de París III - Sorbonne Nouvelle. (Tesis doctoral).

Carbó, Teresa. 1987. ¿Cómo habla el poder legislativo en México? Revista Mexicana de Sociología 49(2). 165-180. Dor: 10.237/3540467.

Clark, Herbert H. 1994. Managing problems in speaking. Speech communication 15(3-4). 243-250.

Clark, Herbert H. \& Fox Tree, Jean. 2002. Using uh and um in spontaneous speaking. Cognition 84(1). 73-111. DOI: 10.1016/s00100277(02)00017-3.

Cribble, Ludivine. 2018. Discourse markers and (dis)fluency. Forms and functions across languages and registers. Ámsterdam/Filadelfia: John Benjamins.

Del Valle, José. 2017. La perspectiva glotopolítica y la normatividad. Anuario de Glotopolítica. 17-39. Editorial Cabiria: Buenos Aires.

Duez, Danielle. 2001. Signification des hésitations dans la production et la perception de la parole spontanée. Revue Parole 17-19. 113-138. Fant, Lars. 1996. Regulación conversacional en la negociación: una comparación entre pautas mexicanas y peninsulares. En Kotschi, Tomás; Oesterreicher, Wulf \& Zimmerman, Klaus. (eds.). El español hablado 
y la cultura oral en España e Hispanoamérica, 147-184. Madrid: Vervuert Iberoamericana.

García, Ofelia. 2009. Bilingual Education in the 21 $1^{\text {st }}$ century: A global perspective. West Sussex: Wiley-Blackwell.

Götz, Sandra. 2013. Fluency in native and nonnative English speech. (Studies in corpus linguistics 53). Ámsterdam: John Benjamins.

Jeong, Hyejeong. 2011. ¿Vacilar? En español y en coreano. En Maurya, Vibha \& Insúa, Mariela (eds). Actas del I congreso ibero-asiático de hispanistas Siglo de Oro e hispanismo general. 327-341. Navarra: GRIso/ Universidad de Navarra.

Jespersen, Otto. 1950 [1924]. The philosophy of grammar. Sexta impresión. Londres: Allen \& Unwin.

Koch, Peter \& Oesterreicher, Wulf. 1990. Lengua hablada en la Romania: español, francés, italiano. (Biblioteca Románica Hispánica) Madrid: Gredos. (Traducción de Araceli López Serena).

Levelt, Willem. 1983. Monitoring and self-repair in speech. Cognition 14(1). 41-104.

Ley de la Comisión de Derechos Humanos del Distrito Federal. 1993. Diario Oficial de la Federación. México: SEGOB.

Ley Orgánica de la Asamblea Legislativa del Distrito Federal. 2008. Gaceta Oficial del Distrito Federal, 19 de diciembre de 2002. Última reforma, 2 de julio de 2014. http://www.aldf.gob.mx/marco-juridico-102-1.html. (Consultado el 20/12/ 2015).

Otheguy, Ricardo; García, Ofelia \& Reid, Wallis. 2015. Clarifying translanguaging and deconstructing named languages: A perspective from linguistics. Applied Linguistics Review 6(3). 281-307. 
Perelman, Chaïm \& Olbrechts-Tyteca, Lucie. 2010 [1969]. The new rhetoric. A treatise on argumentation. Indiana: Universidad de Notre Dame. (Traducción de John Wilkinson \& Purcell Weaver). PRESEEA. Proyecto para el Estudio Sociolingüistico del Español de Espana y de América. 2008. Marcas y etiquetas mínimas obligatorias. Vers. 1.2. 17-02-2008. http://www.linguas.net/preseea. (Consultado el $19 / 12 / 2015)$.

Quesada, Luis B. 2019. Vacilaciones y planificación discursiva en entrevistas de la Asamblea Legislativa del Distrito Federal: la elección de la ombudsperson de derechos humanos en 2013. Boletín de Filología 54(1). 255-282. DoI: 10.4067/S0718-93032019000100255.

Schachter, Paul \& Shopen, Timothy. 2007. Parts-of-speech systems. En Shopen, T. (ed.) Language typology and syntactic description. 1-60. Cambridge: Cambridge University Press.

Vázquez Laslop, María Eugenia. 2011. Elementos del mecanismo de la toma de turnos en el debate parlamentario y alternativas de transcripción. En Martín Butragueño, Pedro (ed.). Realismo en el análisis de corpus orales. Primer Coloquio de Cambio y Variación Lingüistica. Ciudad de México: El Colegio de México.

Watanabe, Michiko; Hirose, Keikichi; Den, Yasuharu \& Minematsu, Nobuaki. 2008. Filled pauses as cues to the complexity of upcoming phrases for native and non-native listeners. Speech Comunication 50(2). 81-94. 